\title{
Influence of fogging lenses and cycloplegia on open-field automatic refraction
}

\author{
Queirós A ${ }^{1}$, J. González-Méijome ${ }^{1}$, Jorge J. ${ }^{1}$ \\ ${ }^{1}$ Department of Physics (Optometry), School of Sciences, University of Minho, Braga, \\ Portugal.
}

Corresponding Author:

António Queirós

Department of Physics (Optometry)

Campus de Gualtar

University of Minho

Tel.: +351253604320

Fax : +351 253678981

e-mail: aqp@fisica.uminho.pt

4710-057 Braga - Portugal 
Influence of fogging lenses and cycloplegia on open-field automatic refraction

\section{ABSTRACT}

Purpose: To compare refractive values measured without cycloplegia, cycloplegia and fogging lenses using an open-field auto-refractor.

Methods: One hundred and forty-two young adults were enrolled from a university population; 96 were female $(67.6 \%)$ and 46 were male (32.4\%), the age range was 18 to 26 years (mean $22.3 \pm 3.7$ years). The refraction measurement was obtained with autorefractor Grand Seiko Auto Ref/Keratometer WAM-5500 (GS) under three conditions, always in this sequence: 1) without cycloplegia (GS), 2) without cycloplegia but using a +2.00D fogging lens (GS_2D) and 3) with cycloplegia (GS_cycl).

Results: When the average values of spherical equivalent were compared both accommodation control strategies were almost equally successful: $\mathrm{GS}, \mathrm{M}=-0.85 \pm 2.21$ D; GC_2D, M= $-0.53 \pm 2.10 \mathrm{D}$ and GS_cycl, $\mathrm{M}=-0.57 \pm 2.24 \mathrm{D}$ (Kruskal-Wallis Test, $\mathrm{p}<0.001)$. When the results were analyzed separately for different refractive groups, emmetropes and hyperopes show statistically significant differences while myopes did not. When both accommodation strategies were compared there was a trend for more myopic subject to display more negative values under cycloplegia, while low myopes, emmetropes and hyperopes tend to display more negative values with the $+2.00 \mathrm{D}$ fogging lenses (less effectiveness of accommodation control).

Conclusions: Over-refraction through $+2.00 \mathrm{D}$ fogging lenses is useful to achieve additional relaxation of the accommodative response in a similar way than cycloplegia when open-field autorefraction is performed in young adults.

Keywords: Open-field autorefraction, fogging lenses, cycloplegia, GrandSeiko. 
Influence of fogging lenses and cycloplegia on open-field automatic refraction

\section{INTRODUCTION}

Objective refraction is an important part of optometric examination. Despite retinoscopy is still today the best starting point for subjective refraction (Jorge et al. 2005a), auto-refraction is widely used by optometrists and ophthalmologist. Different research reports support the reliability (McBrien and Millodot 1985) of these systems particularly when autorefraction is performed under cycloplegia (Chat and Edwards 2001; Jorge et al. 2005b; Choong et al. 2006).

Most closed-field autorefractors have built-in automatic fogging mechanisms to avoid accommodation during measurement. However, the targets used in these instruments stimulate accommodation given more negative values than cycloplegic refraction (Jorge et al. 2005b), which leads to an overestimation of myopia degree and prevalence (Jorge et al. 2006b).

Nevertheless, the use of cycloplegia is limited by the time needed to achieve full effect, its association with patient discomfort, inconvenience, additional cost, risk of increase of intraocular pressure, and limited access to diagnostic drugs to optometrists in certain countries. In Europe, with the exception of Ireland, Netherlands and the U.K., it is forbidden for optometrists to use cycloplegic agents. In addition, most refractive exams in these countries and in the US are also performed without cycloplegia.

Open-field autorefractors were first introduced to the marketplace in the late eighties and the first research reports showed that they significantly overcome those difficulties by observation of real-world targets rather than enclosed viewing of internal fixation target. Even with these instruments, a minimal accommodative response could be expected to affect the final value particularly in children and hyperopes (Chat and Edwards 2001). Gwiazda and Weber compared the spherical equivalent refraction 
Influence of fogging lenses and cycloplegia on open-field automatic refraction

obtained with three different autorefractors without cycloplegia: two open-field autorefractors (Grand Seiko WR-5100K and Canon R-1) and closed-field autorefractor (Nidek ARK-700). Not surprisingly both open-field autorefractors recorded more positive values than the Nidek closed field instrument by a difference of $0.65 \mathrm{D}$ and $0.22 \mathrm{D}$, respectively; in the comparison between both open-field instruments the Grand Seiko gave more positive values by an average of 0.43 D (Gwiazda and Weber 2004). Open field autorefraction also demonstrated to be effective after LASIK surgery showing good agreement between subjective refraction under non-cycloplegic and cycloplegic conditions (Bailey et al. 2005).

Another widely used open-field autorefractor, the Shin-Nippon NVision K-5001 correlated well with subjective refraction; furthermore, subjective refraction gave more myopic or less hyperopic values by an average $0.14 \mathrm{D}$, which suggests a good control of instrumental accommodation stimulus by the instrument (Davies et al. 2003). Markedly different results had been previously found with closed field autorefractors (Nayak et al. 1987).

Thus, whatever the technique used to obtain an objective starting point for subjective refraction, it is important to overcome the effect of accommodation (Suryakumar and Bobier 2003; Zhao et al. 2004; Choong et al. 2006). Apart from cycloplegia, accommodative response can be controlled by using plus lenses. This is a common practice during noncycloplegic retinoscopy. This procedure is referred to as optical fogging and is recognized as a clinical optometric procedure for testing of the pre-school child (Ward and Charman 1987; Ciner 1997). However, the effectiveness of using fogging lenses to perform open-field autorefraction either with or without cycloplegia to avoid accommodative activity is yet to be investigated. This study will 
Influence of fogging lenses and cycloplegia on open-field automatic refraction

help to understand if additional accommodation control can be achieved using fogging lenses and/or cycloplegia during open-field autorefraction.

The purpose of this study was to evaluate objective automatic refraction with an open-field autorefractor without and with accommodation control using plus fogging lenses and cycloplegia. 
Influence of fogging lenses and cycloplegia on open-field automatic refraction

\section{MATERIAL AND METHODS}

One hundred and forty-two young adults, university students, of which 96 were female $(67.6 \%)$ and 46 were male (32.4\%), had been enrolled for this study. Subject's ages ranged from 18 to 26 years old (mean \pm SD: $22.3 \pm 3.7$ years).

In order to analyse separately the potential effect of plus lenses and cycloplegia on accommodation control for different refractive status (emmetropia, myopia and hyperopia), three refractive groups were established according to the spherical equivalent $[\mathrm{M}=$ sphere+(cylinder/2)] as follows: myopia $(\mathrm{M} \leq-0.50 ; \mathrm{n}=38 ; 26.8 \%)$, emmetropia $(-0.50<M<+0.50 ; n=42 ; 29.5 \%)$ and hyperopia $(M \geq+0.50 ; n=62 ; 43.7 \%)$ (Jorge et al. 2007).

After the nature of the study was explained, a consent form was signed by the patient before being enrolled. The research followed the Declaration of Helsinki rules and was reviewed and approved by the Scientific Committee of the School of Sciences of Minho University (Portugal). Inclusion criteria required that the subjects did not suffer from any current eye disease or injury, was not taking any ocular or systemic medication that could affect the accommodative response and had best correct visual acuity of 20/20 or better in each eye. The intraocular pressure was checked with an noncontact tonometer (Nidek Model NT-4000, non-contact tonometer optically) (Queiros et al. 2006).

Autorefraction was measured with an open-field instrument, the Grand Seiko Auto Ref/Keratometer WAM-5500 (Grand Seiko Co., Ltd., Hiroshima, Japan. This instrument is an infrared autorefractor that provides an open binocular field of view, while the patient is looking at a distant fixation target. In its static mode used in this study, the Grand Seiko takes one reading per second. 
Influence of fogging lenses and cycloplegia on open-field automatic refraction

The subject was seated erect with the head stabilized in a chin-rest. The refraction measurement was obtained in three conditions and in this sequence: first without cycloplegia (GS), second, without cycloplegia with the $+2.00 \mathrm{D}$ fogging lenses at $12 \mathrm{~mm}$ from corneal vertex (GS_2D) and finally with cycloplegia (GS_cycl). For each condition three independent readings were taken, converted to vector format and then averaged in order to avoid errors by averaging refractive values in clinical notation. Measurements only from the right eye of each patient were considered. Noncycloplegic refraction was taken first followed by cycloplegic refraction obtained 30 minutes later. Cycloplegia was achieved by instillation of one eyedrop $1 \%$, cyclopentolate (Colircusí Cyclopegic) in each eye twice with a 5-min interval.

Descriptives (mean \pm S.D.) were obtained for the vector components of refraction M, J0 and J45 according to Fourier analysis as recommended by Thibos (Thibos et al. 1997). For comparison purposes, to all the results obtained with the $+2.00 \mathrm{D}$ to fogging lenses this value was subtracted. This value is the equivalent refraction in the ocular plane for a vertex distance of $12 \mathrm{~mm}$.

Kolmogorov-Smirnov Test was produced in order to evaluate normality of data distribution. Mann-Whitney Test was used to compare the measurement between the male and female subjects. Kruskal-Wallis Test was done to evaluate if statistically different values were given by different instruments and different conditions for $\mathrm{M}, \mathrm{J} 0$ and J45. Wilcoxon Signed Ranks Test was used for pairwise comparison between instruments. Graphical regression analysis was also produced to illustrate the relationship between $\mathrm{M}, \mathrm{J} 0$ and $\mathrm{J} 45$ values obtained using different instruments under different conditions. 
Influence of fogging lenses and cycloplegia on open-field automatic refraction

The statistical procedures used to compare results among different instruments and conditions, were described by Bland and Altman (Bland and Altman 1986). The $95 \%$ limits of agreement (mean of the difference $\pm 1.96 *$ S.D. of the difference) were also calculated. This type of analysis makes it easier to assess the level of agreement between techniques, spot outliers and see whether there is any trend in bias. SPSS statistical package v.15 (SPSS Inc., Chicago, IL, USA) was used. 
Influence of fogging lenses and cycloplegia on open-field automatic refraction

\section{$\underline{\text { RESULTS }}$}

The mean objective refraction for the whole sample, as per the autorefractor with cycloplegic (GS_cycl), ranged from -9.00 to + 2.25 D; mean spherical equivalent was $0.57 \pm 2.24 \mathrm{D}$ (mean \pm S.D.) with the maximum amount of astigmatism being $-3.25 \mathrm{D}$. According to the refractive classification, 26.8\% $(n=38)$ of the sample were myopes, $29.5 \%(n=42)$ were emmetropes and $43.7 \%(n=62)$ were hyperopes.

Table 1 presents descriptive results and statistical comparison of the refractive components M, J0 and J45 between instruments and conditions. Statistically significant differences were found for the spherical equivalent $(\mathrm{p}<0.001, \mathrm{~K}-\mathrm{W}$ Test). The same analysis was repeated by splitting the sample into the three refractive groups. Again, the spherical equivalent showed statistically significant differences between the three methods but only for emmetropes and hyperopes. The astigmatic component $\mathrm{J} 45$ also showed statistically significant differences between methods for hyperopic group.

Table 2 presents the pairwise comparison between different refractive methods. For the spherical equivalent there was statistically significant differences between autorefraction without fogging lenses or cycloplegic (GS) and refraction over the +2.00 D lenses (GS_2D); mean difference was -0.32 D with GS being more negative than GS_2D ( $<<0.001$, Wilcoxon Signed Ranks Test). Graphical analysis of difference against means is shown in figure 1. The narrower limits of agreement were presented for $\mathrm{J} 45$ followed by $\mathrm{J} 0$ and the largest interval was for the comparison of spherical equivalents. Surprisingly, the two conditions where the accommodation was supposed to be under control display the largest limits of agreement, but the least spread around the regression line (highest correlation coefficient). The trend of GS_2D to render more positive or less negative values (higher hyperopic shift) is more evident for moderate 
and high myopes and this trend was statistically significant. Again, when comparing GS_2D with GS_cycl a higher hyperopic shift was observed with GS_2D in for moderate and high myopes.

When cycloplegic was used (GS_cycl) mean difference with GS was -0.28 D; in this case the difference only approaches statistical significance $(\mathrm{p}=0.064)$, however the average value has the same clinical meaning as that previously obtained in the comparison of GS with GS_2D, with both values being above \pm 0.25 D. Conversely, there was not any difference between the two conditions that control the accommodative response, GS_2D vs GS_cycl ( $\mathrm{p}>0.05)$.

Regarding the astigmatic components there was only statistically significant differences between GS and GS_2D for the J0 component (mean difference -0.02 D; $\mathrm{p}<0.001)$. 
Influence of fogging lenses and cycloplegia on open-field automatic refraction

\section{DISCUSSION}

In this study, the used of fogging lenses was used in order to evaluate if an addition effect on accommodation control can be achieved in open-field autorefractors using a fogging lens instead of cycloplegia. Our results show that the average spherical equivalent refraction in such circumstances is very similar to that obtained under cycloplegia, while both situations (cycloplegia or fogging lenses) give more positive or less negative values than open-field autorefraction with no additional control of accommodative response. Considering that more positive spherical equivalent indicates less accommodative activity present results show that open-field autorefractor itself was not totally effective showing more negative or less positive values than the remaining two measures. As expected, this effect of myopic shift was not statistically significant for myopes, despite the $0.40 \mathrm{D}$ difference between fogging lens method and regular autorefraction (GS). Although this result could be expect based on the less active accommodative system in myopes, the larger variability of refraction in this group could also account for the lack of statistical significance. Conversely, emmetropic and hyperopic groups displayed significant evidence that fogging lenses method was as effective as cycloplegia to prevent myopic shift. Fogging lenses seems to be effective for emmetropes, cycloplegia was more effective for hyperopes; however, differences between both methods were $0.15 \mathrm{D}$ and $0.16 \mathrm{D}$, respectively. This result could be expected based on the more active accommodation in hyperopes.

Some studies suggest that noncycloplegic autorefraction has reasonable accuracy and repeatability when compared with noncycloplegic retinoscopy and subjective refraction (Jorge et al. 2005a). However, in that study, which compared three techniques of refraction without the use of a cycloplegic agent: closed field 
Influence of fogging lenses and cycloplegia on open-field automatic refraction

autorefraction, retinoscopy and subjective refraction, noncycloplegic autorefraction displayed more negative values of spherical equivalent refraction by $0.45 \mathrm{D}$, and this was clinically significant. Myopic shift induced under certain clinical conditions and instruments is a concern particularly in hyperopic patients and it's a phenomenon common to autorefraction (McCaghrey and Matthews 1993; Bullimore et al. 1998; Gwiazda and Weber 2004; Jorge et al. 2005b) and other modern devices such as clinical aberrometers (Cervino et al. 2006).

Direct comparison of values obtained from the three refractive methods can only be compared by subtracting $2.00 \mathrm{D}$ to all refractive values obtained with the fogging lens in place, in order to discard the optical effect induced by the fogging lenses in the spectacle-eye system. This avoids the systematic error induced by over refraction with autorefractors (Kimura et al. 2007). Ward and Charman observed that above a value equal or higher than 2D of fogging, it is expected that the accommodative system returns to its resting position in a small group of adult myopes and hyperopes (Ward and Charman 1987).

Differences in agreement between methods for the three refractive groups are evident in the graphical analysis presented in figure 1. As a consequence, when both methods of accommodation control were compared, fogging lenses gave more positive values of refraction than cycloplegic refraction, while a significant trend towards the opposite effect was observed for emmetropes and hyperopes. Compared to regular open-field autorefraction the use of fogging lenses was effective to control the accommodative response, particularly for moderate and high myopes $(\leq-2.00 \mathrm{D})$, and less effectively for emmetropes and hyperopes. Despite this, when fogging lenses were used, 90\% (128/142) of the sample show a good control of accommodative response 
(GS_2D more positive than GS, difference between methods $>0$ ), which was quite similar to $93 \%(132 / 142)$ that could be achieved with cycloplegia. One curious feature of the results is the fact that blurring high myopes moves refractive error in a more positive direction than cycloplegia. We cannot explain this finding at present but we can argue that increased spherical aberration in the myopic eyes which becomes more important when the pupil is dilated could account for this effect of overestimation of myopia under cycloplegia (Benjamin 2006).An underestimation of myopia with the fogging lens is not discarded but the first possibility is more likely. This subject disserves further investigation.

In summary, the present study has demonstrated that the use of fogging lenses can be effective to control undesirable accommodative response while performing autorefraction, adding an important relaxation effect to the already reduced accommodative stimulus which is an important advantage of open-field autorefraction in young adults. Other potential application of this methodology is on nonpharmacological accommodative control in massive screening of pediatric populations. Although the present study did not tested this hypothesis, it is expected that open-field autorefraction in addition to optical fogging by using plus lenses in front of the eye while performing autorefraction in children, will provide more reliable values than regular open-field autorefraction itself. Fotedar et al. demonstrated that in the absence of cycloplegia as much as $17.8 \%$ of 12 year-old children will be misclassified as myopic (Fotedar et al. 2007). If the effectiveness of fogging lenses to avoid accommodative stimulus in children will be proven, the use of cycloplegic could be avoided in large scale screening without risk of myopia overestimation. The impact of such an approach in the actual incidence of refractive error is also of enormous importance, (Jorge et al. 
Influence of fogging lenses and cycloplegia on open-field automatic refraction

2006a; Ferrer-Blasco et al. 2007) particularly regarding the overestimation of myopia prevalence worldwide in many studies conducted without cycloplegic agent or whatever other control of accommodative response. In addition to the absence of adverse response from the administration of the cycloplegic, this methodology could be used by non-medical staff and optometrists not allowed to use diagnostic drugs.

Acknowledgements: The authors wish to thank Socióptica,Lda and Mr. Luis Garcia for the loan of the Grand Seiko Auto Ref/Keratometer WAM-5500. None of the authors has a commercial or financial interest in the instruments presented here. 


\title{
REFERENCES
}

\author{
Reference
}

Bailey, M. D., Twa, M. D., Mitchell, G. L., Dhaliwal, D. K., Jones, L. A., and McMahon, T. T. (2005). Repeatability of autorefraction and axial length measurements after laser in situ keratomileusis. J Cataract Refract Surg 31, 1025-1034.

Benjamin, W. J. (2006). Monocular and Binocular Subjective Refraction. In 'Borish Clinical Refraction, 2nd edition'. pp. 790-872. (Butterworth-Heinemann: St. Louis.)

Bland, J. M. and Altman, D. G. (1986). Statistical methods for assessing agreement between two methods of clinical measurement. Lancet 1, 307-310.

Bullimore, M. A., Fusaro, R. E., and Adams, C. W. (1998). The repeatability of automated and clinician refraction. Optom Vis.Sci. 75, 617-622.

Cervino, A., Hosking, S. L., Rai, G. K., Naroo, S. A., and Gilmartin, B. (2006). Wavefront analyzers induce instrument myopia. J Refract.Surg. 22, 795-803.

Chat, S. W. and Edwards, M. H. (2001). Clinical evaluation of the Shin-Nippon SRW-5000 autorefractor in children. Ophthalmic Physiol Opt. 21, 87-100.

Choong, Y. F., Chen, A. H., and Goh, P. P. (2006). A comparison of autorefraction and subjective refraction with and without cycloplegia in primary school children. Am J Ophthalmol 142, 68-74.

Ciner, E. (1997). Refractive error in young children. In 'Eye Care for Infants and Young Children'. (Ed. Moore BD.) pp. 47-73. (Butterworth-Heinemann: Boston.)

Davies, L. N., Mallen, E. A., Wolffsohn, J. S., and Gilmartin, B. (2003). Clinical evaluation of the Shin-Nippon NVision-K 5001/Grand Seiko WR-5100K autorefractor. Optom Vis.Sci 80, 320-324.

Ferrer-Blasco, T., Gonzalez-Meijome, J. M., and Montes-Mico, R. (2007). Agerelated changes in the human visual system and prevalence of refractive conditions in patients attending an eye clinic. $J$ Cataract Refract Surg (in press).

Fotedar, R., Rochtchina, E., Morgan, I., Wang, J. J., Mitchell, P., and Rose, K. A. (2007). Necessity of cycloplegia for assessing refractive error in 12-year-old children: a population-based study. Am.J Ophthalmol 144, 307-309.

Gwiazda, J. and Weber, C. (2004). Comparison of spherical equivalent refraction and astigmatism measured with three different models of autorefractors. Optom.Vis.Sci. 81, 56-61.

Jorge, J., Almeida, J. B., and Parafita, M. A. (2006a). What is the real prevalence of myopia? Ophthalmic Physiol Opt. 26, 31. 
Jorge, J., Almeida, J. B., and Parafita, M. A. (2007). Refractive, biometric and topographic changes among Portuguese university science students: a 3-year longitudinal study. Ophthalmic Physiol Opt 27, 287-294.

Jorge, J., Gonzalez-Meijome, J. M., Queiros, A., Almeida, J. B., and Parafita, M. A. (2006b). Differences in ametropia prevalence obtained with different methods of refraction. Acta Ophthalmol Scand. 84, 165.

Jorge, J., Queiros, A., Almeida, J. B., and Parafita, M. A. (2005a).

Retinoscopy/Autorefraction: which is the best starting point for a noncycloplegic refraction? Optom Vis.Sci. 82, 64-68.

Jorge, J., Queiros, A., Gonzalez-Meijome, J., Fernandes, P., Almeida, J. B., and Parafita, M. A. (2005b). The influence of cycloplegia in objective refraction. Ophthalmic Physiol Opt 25, 340-345.

Kimura, S., Hasebe, S., and Ohtsuki, H. (2007). Systematic measurement errors involved in over-refraction using an autorefractor (Grand-Seiko WV-500): is measurement of accommodative lag through spectacle lenses valid? Ophthalmic Physiol Opt. 27, 281-286.

Mallen, E. A., Wolffsohn, J. S., Gilmartin, B., and Tsujimura, S. (2001). Clinical evaluation of the Shin-Nippon SRW-5000 autorefractor in adults. Ophthalmic Physiol Opt. 21, 101-107.

McBrien, N. A. and Millodot, M. (1985). Clinical evaluation of the Canon Autoref R-1. Am.J.Optom.Physiol Opt. 62, 786-792.

McCaghrey, G. E. and Matthews, F. E. (1993). Clinical evaluation of a range of autorefractors. Ophthalmic Physiol Opt. 13, 129-137.

Nayak, B. K., Ghose, S., and Singh, J. P. (1987). A comparison of cycloplegic and manifest refractions on the NR-1000F (an objective Auto Refractometer).

Br.J.Ophthalmol. 71, 73-75.

Queiros, A., Gonzalez-Meijome, J. M., Fernandes, P., Jorge, J., Almeida, J. B., and Parafita, M. A. (2006). Non-contact tonometry synchronized with cardiac rhythm and its relationship with blood pressure. Ophthalmic Physiol Opt 26, 384-391.

Suryakumar, R. and Bobier, W. R. (2003). The manifestation of noncycloplegic refractive state in pre-school children is dependent on autorefractor design. Optom.Vis.Sci. 80, 578-586.

Thibos, L. N., Wheeler, W., and Horner, D. (1997). Power vectors: an application of Fourier analysis to the description and statistical analysis of refractive error. Optom Vis.Sci 74, 367-375.

Ward, P. A. and Charman, W. N. (1987). An objective assessment of the effect of fogging on accommodation. Am.J.Optom.Physiol Opt. 64, 762-767.

Zhao, J., Mao, J., Luo, R., Li, F., Pokharel, G. P., and Ellwein, L. B. (2004). Accuracy of noncycloplegic autorefraction in school-age children in China. Optom.Vis.Sci. 81, 49-55. 
Influence of fogging lenses and cycloplegia on open-field automatic refraction 
Influence of fogging lenses and cycloplegia on open-field automatic refraction

Table 1. Mean refractive error (mean \pm S.D.) obtained of whole sample and individual groups refractive for $\mathrm{M}, \mathrm{J} 0$ and $\mathrm{J} 45$ components of under different conditions: autorefractor GS, GS_2D and GS_cycl (n=142, values are expressed in diopters).

\begin{tabular}{|c|c|c|c|c|c|c|}
\hline & & $\mathbf{N}$ & $\begin{array}{c}\text { GS } \\
\text { (Mean } \pm \text { S.D.) }\end{array}$ & $\begin{array}{c}\text { GS_2D } \\
\text { (Mean } \pm \text { S.D. })\end{array}$ & $\begin{array}{c}\text { GS_cycl } \\
\text { (Mean } \pm \text { S.D.) }\end{array}$ & $p$ \\
\hline $\mathrm{M}$ & & 142 & $-0.85 \pm 2.21$ & $-0.53 \pm 2.10$ & $-0.57 \pm 2.24$ & $<0.001$ \\
\hline JO & Whole sample & 142 & $+0.13 \pm 0.30$ & $+0.15 \pm 0.29$ & $+0.13 \pm 0.30$ & 0.605 \\
\hline J45 & & 142 & $+0.01 \pm 0.23$ & $-0.01 \pm 0.22$ & $0.02 \pm 0.25$ & 0.117 \\
\hline \multirow{3}{*}{ M } & Myopia & 38 & $-3.77 \pm 2.51$ & $-3.37 \pm 2.25$ & $-3.59 \pm 2.41$ & 0.736 \\
\hline & Emmetropic & 42 & $-0.05 \pm 0.33$ & $+0.25 \pm 0.40$ & $+0.10 \pm 0.26$ & $<0.001$ \\
\hline & Hyperopic & 62 & $+0.39 \pm 0.33$ & $+0.67 \pm 0.30$ & $+0.83 \pm 0.27$ & $<0.001$ \\
\hline \multirow{3}{*}{ JO } & Myopia & 38 & $+0.23 \pm 0.41$ & $+0.24 \pm 0.36$ & $+0.25 \pm 0.41$ & 0.752 \\
\hline & Emmetropic & 42 & $+0.06 \pm 0.24$ & $+0.06 \pm 0.23$ & $+0.09 \pm 0.24$ & 0.846 \\
\hline & Hyperopic & 62 & $+0.11 \pm 0.24$ & $+0.16 \pm 0.26$ & $+0.08 \pm 0.25$ & 0.300 \\
\hline \multirow{3}{*}{ J45 } & Myopia & 38 & $-0.00 \pm 0.40$ & $+0.02 \pm 0.37$ & $-0.01 \pm 0.43$ & 0.982 \\
\hline & Emmetropic & 42 & $+0.00 \pm 0.13$ & $-0.01 \pm 0.12$ & $+0.00 \pm 0.11$ & 0.876 \\
\hline & Hyperopic & 62 & $+0.01 \pm 0.13$ & $-0.02 \pm 0.15$ & $+0.04 \pm 0.16$ & 0.007 \\
\hline
\end{tabular}

$\mathrm{N}$, number of subjects; GS, autorefraction noncycloplegic; GS_2D, autorefraction with fogging lenses $+2.00 \mathrm{D}$ noncycloplegic; GS_cycl, autorefraction with cycloplegic. Comparison among three conditions (Kruskal-Wallis Test). 
Influence of fogging lenses and cycloplegia on open-field automatic refraction

Table 2. Mean difference, S.D. and significance level and 95\% limits of agreement for the components M, J0 and J45 for the GS, GS_2D and GS_cycl.

\begin{tabular}{|c|c|c|c|c|}
\hline & \multirow{2}{*}{ Mean \pm S.D. } & \multirow{2}{*}{ Sig. * } & \multicolumn{2}{|c|}{ Limits of agreement } \\
\hline & & & Mean $+1.96 \times$ S.D. & Mean - $1.96 \times$ S.D. \\
\hline \multicolumn{5}{|c|}{ Autorefraction GS vs Autorefraction GS with fogging +2.00D } \\
\hline M & $-0.32 \pm 0.32$ & $<0.001$ & 0.31 & -0.94 \\
\hline J0 & $-0.02 \pm 0.14$ & $<0.001$ & 0.25 & -0.29 \\
\hline $\mathrm{J} 45$ & $+0.01 \pm 0.11$ & 0.230 & 0.23 & -0.20 \\
\hline \multicolumn{5}{|c|}{ Autorefraction GS vs Autorefraction GS_cycl } \\
\hline M & $-0.28 \pm 0.29$ & 0.064 & 0.29 & -0.85 \\
\hline J0 & $+0.00 \pm 0.16$ & 0.299 & 0.32 & -0.31 \\
\hline $\mathrm{J} 45$ & $-0.01 \pm 0.11$ & 0.228 & 0.21 & -0.23 \\
\hline \multicolumn{5}{|c|}{ Autorefraction GS with fogging +2.00D vs Autorefraction GS_cycl } \\
\hline M & $+0.04 \pm 0.34$ & 0.495 & 0.70 & -0.63 \\
\hline J0 & $+0.02 \pm 0.19$ & 0.094 & 0.40 & -0.36 \\
\hline $\mathrm{J} 45$ & $-0.03 \pm 0.13$ & 0.050 & 0.23 & -0.28 \\
\hline
\end{tabular}

$\left(^{\star}\right)$ Wilcoxon Signed Ranks Test 
Figure 1. Plots of difference vs mean of $M$ component for different comparisons GSvsGS_2D (A), GSvsGS_cycl (B) and GS_2DvsGS_cycl (C).
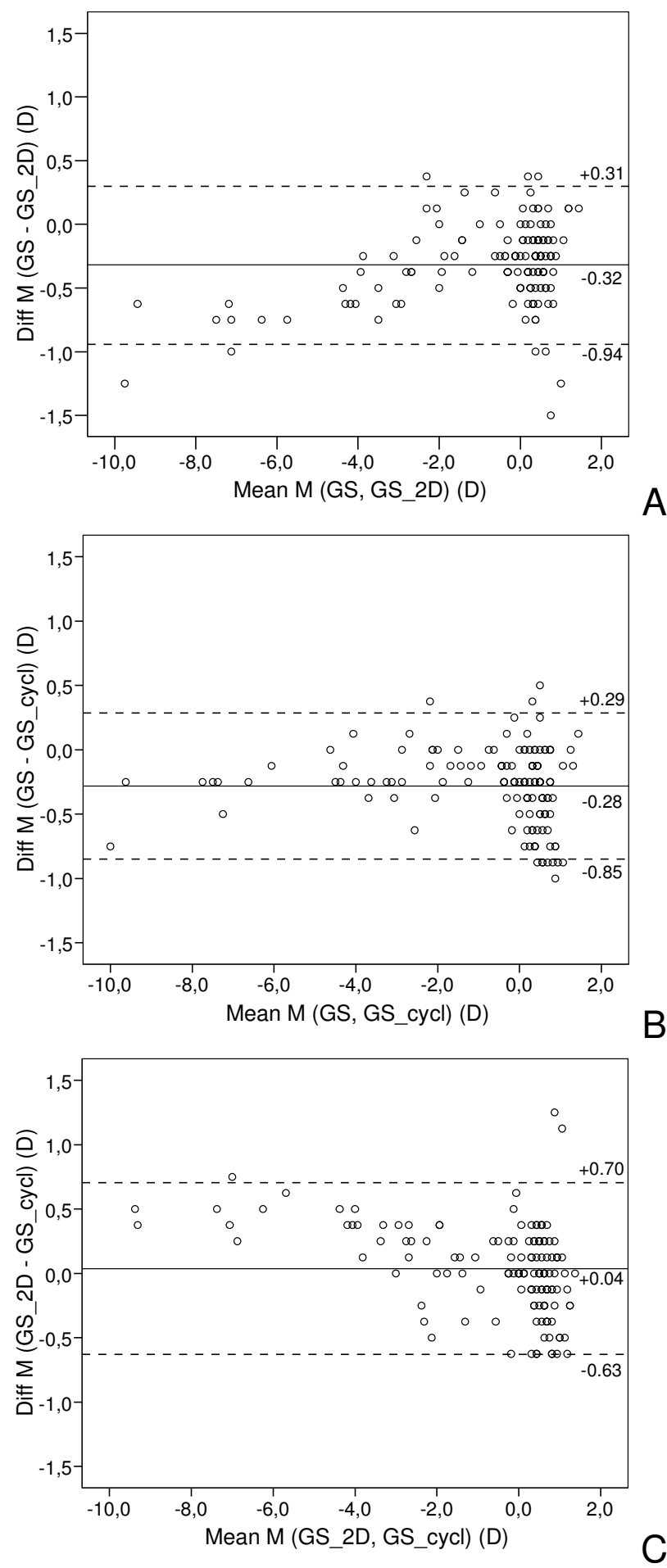